\title{
Repeated exposure to sevoflurane in neonatal rats impairs cognition in adulthood via the PKA-CREB-BDNF signaling pathway
}

\author{
JILI ZHAO ${ }^{1}$, JINYU REN ${ }^{2}$, SHUANG LIU ${ }^{3}$, YANAN GONG ${ }^{4}$, \\ PING MENG ${ }^{5}$, HAITAO TAN $^{6}$ and YONGGANG $\mathrm{CHEN}^{7}$
}

\begin{abstract}
${ }^{1}$ Department of Anesthesiology, Zhangqiu District Maternal and Child Health Care Hospital, Jinan, Shandong 250200; ${ }^{2}$ Department of Anesthesiology, The Third Hospital of Jinan, Jinan, Shandong 250032;

${ }^{3}$ Department of Operating Room, Zhangqiu District Maternal and Child Health Care Hospital; Departments of ${ }^{4}$ Cardiovascular Medicine and ${ }^{5}$ Burn and Plastic Surgery, The People's Hospital of Zhangqiu Area, Jinan, Shandong 250200; ${ }^{6}$ Department of Anesthesiology, Jining No. 1 People's Hospital, Jining, Shandong 272011;

${ }^{7}$ Department of Anesthesiology, People's Hospital of Gaomi, Gaomi, Shandong 261500, P.R. China
\end{abstract}

Received February 13, 2020; Accepted December 3, 2020

DOI: $10.3892 / \mathrm{etm} .2021 .10877$

\begin{abstract}
Sevoflurane (Sev) anesthesia is widely used in pediatrics due to its low blood-gas partition coefficient and lack of pungency. However, Sev treatment may lead to cognitive dysfunction in later life. The current study administered Sev to neonatal rats to investigate the effects of Sev treatment on cognitive performance in adulthood. In total, 6-day-old rats received $3 \% \mathrm{Sev}$ for $2 \mathrm{~h}$ daily for 3 consecutive days. The cognitive function of rats in adulthood was evaluated in 56-day-old rats by Morris water maze test. The hippocampal neuron morphology was observed by Nissl staining. Hippocampal brain-derived neurotrophic factor (BDNF) levels were measured by ELISA. The protein expression of protein kinase A (PKA), cAMP response element binding protein (CREB), phosphorylated-CREB (p-CREB) and BDNF in hippocampus were assessed by western blotting. The water maze results demonstrated that neonatal treatment with Sev resulted in a significant impairment of cognition in 56-day-old adult rats. Behavioral analysis revealed that $\mathrm{Sev}$ treatment increased latency to first pass the platform and decreased residence in target quadrants and across platform frequency compared with the control group in Morris water maze tests. Furthermore, compared with the control group, neonatal exposure to $\mathrm{Sev}$ reduced the number of neurons and the concentration of BDNF in the hippocampus, a brain region
\end{abstract}

Correspondence to: Dr Yonggang Chen, Department of Anesthesiology, People's Hospital of Gaomi, 77 Zhenfu Street, Gaomi, Shandong 261500, P.R. China

E-mail: ygchen0912@sina.com

Key words: sevoflurane, neuron, cognition, repeated exposure, protein kinase A/cAMP response element binding protein/brain-derived neurotrophic factor important for learning and memory. Additionally, Sev significantly decreased the expression of PKA, p-CREB, BDNF and the $\mathrm{p}-\mathrm{CREB} / \mathrm{CREB}$ ratio. Treatment with bucladesine, a selective PKA agonist, partially reversed the deleterious effects of Sev. In summary, the results indicated that PKA-CREB-BDNF signaling served an important role in the cognitive decline caused by neonatal exposure to Sev.

\section{Introduction}

Sevoflurane (Sev), a common inhaled anesthetic in pediatrics, has the advantages of low airway irritation and a low blood gas distribution coefficient $(1,2)$. Despite these advantages, reports have indicated that Sev significantly increased the incidence of learning deficits in adolescents $(3,4)$. In neonatal animals, Sev induced neurological impairment, including cognitive decline and abnormal social behaviors in adulthood $(5,6)$. Unfortunately, few interventions or treatments prevent these neurological defects (7).

Brain-derived neurotrophic factor (BDNF) servesan important role in neuronal survival, growth and differentiation (8). BDNF is located in the hippocampus, cerebral cortex and basal forebrain, which are critical areas for learning and memory (9). cAMP response element binding protein (CREB) regulates the expression of several genes, including BDNF, that promote synapse formation and neural plasticity $(10,11)$. Furthermore, there are several CREB binding sites in the promoter region of the BDNF gene (12). CREB phosphorylation is essential for its function (13).

Several protein kinases, including protein kinase A (PKA), extracellular receptor kinase and phosphatidylinositol-3 kinases, are known to phosphorylate and activate CREB $(14,15)$. Since activation of PKA/CREB/BDNF signaling is closely associated with memory formation, the current study investigated whether Sev influences cognition via the PKA/CREB/BDNF pathway in the hippocampus in vivo. The present study tested the hypothesis that 
intraperitoneal injections of bucladesine (Buc; also called DB-cAMP), a membrane permeable selective activator of PKA, can cause an improvement in cognition.

\section{Materials and methods}

Materials. The following anesthetics, substances and kits were used: sevoflurane (Sev; Abbott $\mathrm{GmbH}$ ), Buc (Abbott $\mathrm{GmbH}$ ), anti-PKA (cat. no. ab5815; Abcam), anti-CREB (cat. no. ab31387; Abcam), anti-phosphorylated (p-) CREB (cat. no. ab10564; Abcam), anti-BDNF (cat. no. ab226843; Abcam), anti-actin (cat. no. ab179467; Abcam), goat anti-rabbit IgG H\&L (HRP conjugated; cat. no. ab205718; Abcam) and BDNF ELISA kits (cat. no. NI-0035; Beijing North Institute of Biotechnology Co., Ltd.).

Animals. A total of 30 Sprague-Dawley rats (male, 10; female, 20; weight, $220 \pm 20 \mathrm{~g}$ ), 10-12 weeks old, were used. Animals were purchased from Jinfeng Laboratory Animal Co., Ltd. Animals were housed with free access to food and water at a temperature of $22 \pm 2^{\circ} \mathrm{C}$ and $55 \pm 5 \%$ humidity with 12 -h light/dark cycles. Male and female rats were caged at a ratio of 1:2. Female rats were housed in individual cages after they were confirmed to be pregnant until they delivered naturally. Day of birth was noted as postnatal day 0 (P0). The experimental protocol was approved by the Institutional Animal Care and Use Committee of Zhangqiu District Maternal and Child Health Care Hospital (Jinan China).

Anesthetic exposure. In total,55 P6-P8 rat pups (male, 28; female, 27) were placed in a glass chamber $(20 \times 12 \times 10 \mathrm{~cm})$ and rested in a water bath to maintain a constant environmental temperature of $37-38^{\circ} \mathrm{C}$. The Con group (male, 8 ; female, 7) was not exposed to Sev. The Sevgroup (male, 10; female, 10) were exposed to $3 \% \mathrm{Sev}$ in $30 \%$ oxygen carrier gas (in balance with nitrogen) and exposed to $2 \mathrm{~h}$ daily for 3 consecutive days in the chamber (16). Following anesthesia, pups were allowed to recover and were returned to their mothers. For the intervention studies, Buc, a selective PKA agonist (17), was administered to the rats via an intraperitoneal injection at a concentration of $300 \mathrm{nM} / \mathrm{rat} 2 \mathrm{~h}$ prior to $\mathrm{Sev}$ anesthesia (male, 10; female, 10) (18). The Con group did not undergo Buc intraperitoneal injection. All the experiments performed were blinded. At P50, rats underwent behavioral tests. Study protocol is presented in Fig. 1.

Water maze. Three groups of animals were tested for water maze: Con $(n=10), \operatorname{Sev}(n=10)$ and Buc $+\operatorname{Sev}(n=10)$. Rats were trained in an open water maze (diameter, $1.5 \mathrm{~m}$ ) filled with water (temperature, $26^{\circ} \mathrm{C}$ ) made opaque with latex liquid. Prominent extra-maze visual cues around the room remained in fixed positions throughout the experiments. In the behavioral tests, rats were required to locate a hidden submerged platform (diameter, $10 \mathrm{~cm} ; 1.5 \mathrm{~cm}$ below the surface), which remained in the same position for individual animals; Four equally spaced points (north, south, east and west) at the edge of the pool were used as starting positions. The whole process was divided into two parts: the first 4 days were training tests and day 5 was the probe test. During the training tests, trials began when rats were placed in the pool
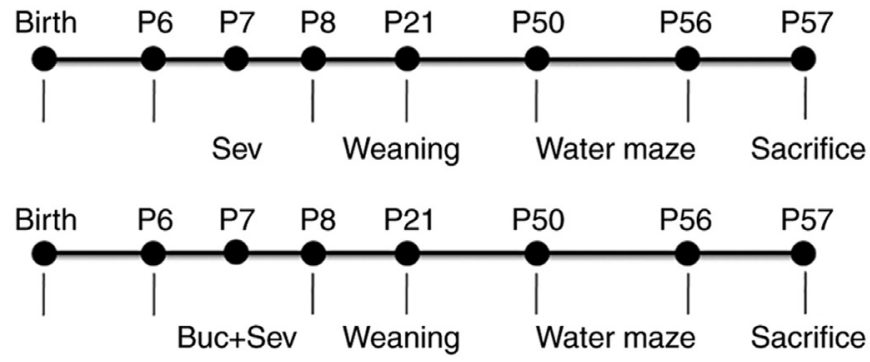

Figure 1. Study protocol of experiment. P, postnatal day; Sev, sevoflurane; Buc, bucladesine.

facing the side wall at a start position and ended once the rats found the platform. If rats had not found the platform within $120 \mathrm{sec}$, they were guided there by hand. In the probe tests, the swimming duration was set to not exceed $120 \mathrm{sec}$. If the rat did not find the platform in $120 \mathrm{sec}$, the task was considered a failure. A Morris water maze video analysis system was purchased from Chinese Academy of Medical Sciences. A video camera was mounted to the ceiling directly above the center of the maze was used in conjunction with the animal tracking system (19).

Nissl staining. Rats were deeply anesthetized with pentobarbital sodium (Shanghai Chemical Reagent Company) at the dose of $50 \mathrm{mg} / \mathrm{kg}$ and perfused transcardially with $0.9 \%$ NaClfor 5 min. Rats were then perfused with $4 \%$ paraformaldehyde (PFA) in $0.1 \mathrm{M}$ phosphate buffer (pH 7.4) for $20 \mathrm{~min}$. Brains were removed, fixed in 4\% PFA for 7 days at room temperature and embedded in paraffin. Following this, $3 \mu \mathrm{M}$-thick paraffin sections were excised from the dentate gyrus (DG) of the hippocampus and Nissl staining $\left(56^{\circ} \mathrm{C}\right.$ for $30 \mathrm{~min}$ ) was successfully optimized onto paraffin sections. Morphological changes of neurons were detected using a light microscope (magnifications, $\mathrm{x} 40, \mathrm{x} 100$ and $\mathrm{x} 200$ ).

Measurement of BDNF levels. Rats were decapitated and sacrificed following $50 \mathrm{mg} / \mathrm{kg}$ pentobarbital sodium anesthesia via intraperitoneal injections. The hippocampus were removed,homogenized and centrifuged at $10,000 \mathrm{x}$ g at $4^{\circ} \mathrm{C}$ for $10 \mathrm{~min}$ to obtain hippocampal homogenates. BDNF levels in hippocampal homogenates were measured using commercially available BDNF ELISA kits (cat. no. NI-1200; Beijing North Institute of Biotechnology Co., Ltd.).

Western blotting. Hippocampus were rinsed twice with cold PBS and dissolved on ice with a RIPA buffer containing $1 \mathrm{Mm}$ Phenylmethanesulfonyl fluoride (Promega Corporation) for $30 \mathrm{~min}$, followed by centrifugation at $12,000 \mathrm{x} \mathrm{g}$ for $10 \mathrm{~min}$ at $4^{\circ} \mathrm{C}$. Protein concentrations were determinate using the BCA protein assay. Total proteins $(50 \mu \mathrm{g} / \mathrm{well})$ were separated via 10\% SDS-PAGE and transferred via electrophoresis onto PVDF membranes. Membranes were blocked with $5 \%$ skimmed milk at room temperature for $1 \mathrm{~h}$ and incubated overnight at $4^{\circ} \mathrm{C}$ with anti-PKA $(1: 1,500)$, anti-CREB (1:600), anti-phosphorylated-CREB (p-CREB; 1:1,000), anti-BDNF $(1: 1,200)$ and anti-actin $(1: 3,000)$. Actin was used as the loading control. Subsequently, membranes were incubated with corresponding secondary antibodies $(1: 1,000)$ 
A

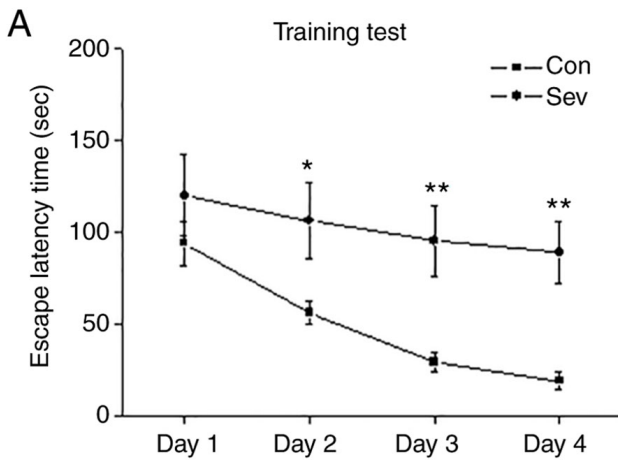

C

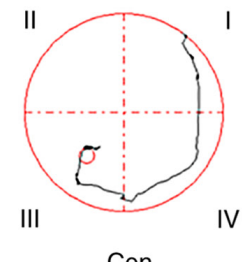

Probe test

E

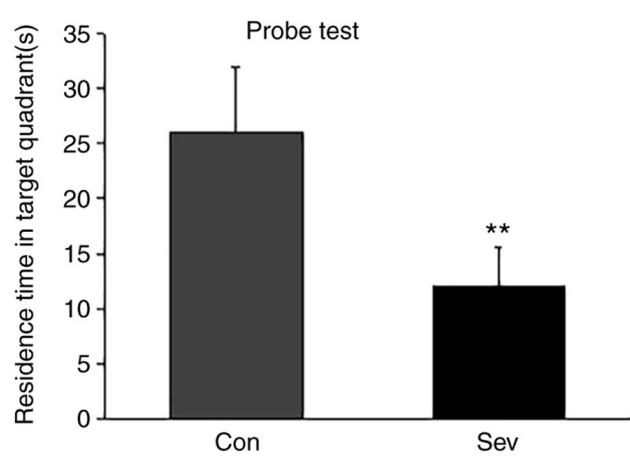

B

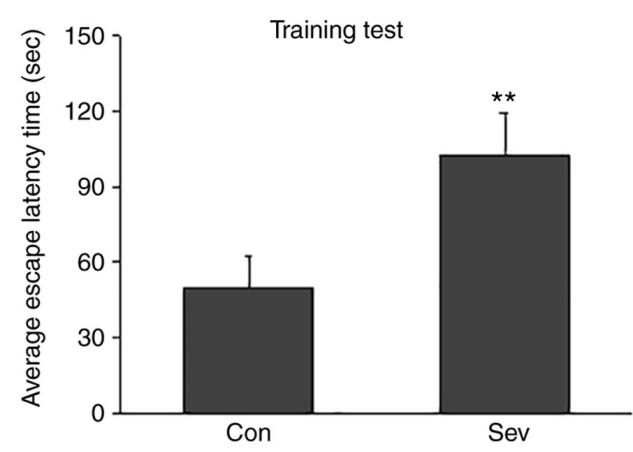

D

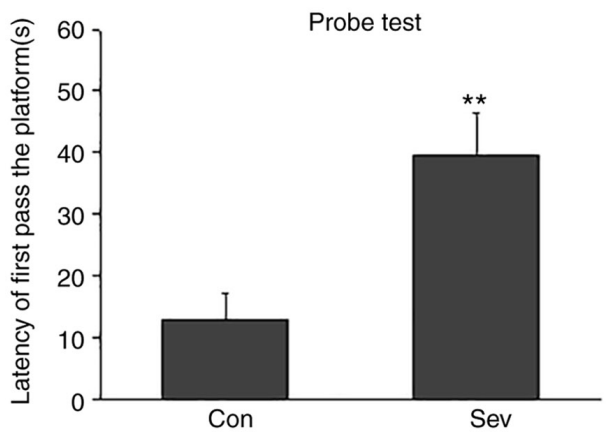

F

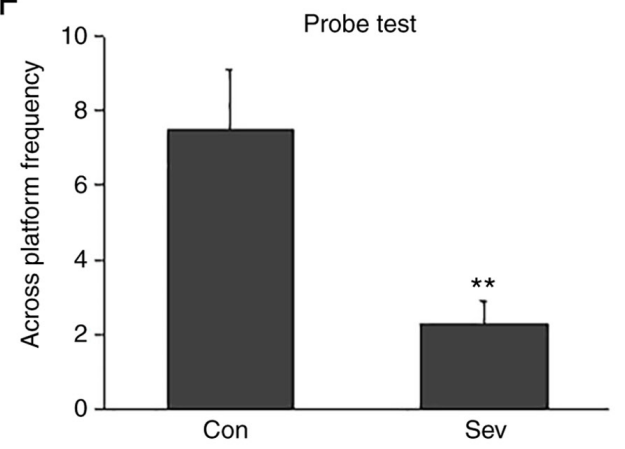

Figure 2. Effect of repeated neonatal exposure to Sev on learning and memory in adult rats examined by Morris water maze tests. (A) Individual and (B) average escape latency times during the training tests. (C) Motion trails in the control and Sev groups during the probe tests. (D) Latency to first pass the platform during the probe tests. (E) Residence time in target quadrant during the probe tests. (F) Across platform frequency during the probe tests. $n=10 / g r o u p$. ${ }^{*} \mathrm{P}<0.05 ;{ }^{* *} \mathrm{P}<0.01$ vs. Con. Sev, sevoflurane; Con, control.

at $37^{\circ} \mathrm{C}$ for $2 \mathrm{~h}$ and reactions were visualized with chemiluminescence reagents provided by an ECL kit (Bioworld Technology, Inc.) and exposed to X-ray film. Blot intensities were quantified with densitometry by Quantity One v4.6.6 (Bio-Rad Laboratories).

Statistical analysis. Paired T-tests and one-way ANOVA followed by Bonferroni's correction were performed to compare differences between groups. SAS software (Wuhan Oriental Seth Software Co., Ltd.) was used for statistical analysis. Data are presented as mean \pm standard error of the mean. $\mathrm{P}<0.05$ was considered to indicate a statistically significant difference.

\section{Results}

Sev induces cognitive impairment. Morris water maze tests were used to evaluate the effect of Sev on cognition. In the training tests, escape latency times were significantly increased following repeated exposure to $\mathrm{Sev}$ (Fig. 2A and B). In the probe tests, Sev significantly increased latency to first pass the platform (Fig. 2C and D) and decreased residence times in target quadrants (Fig. 2E) and across platform frequencies (Fig. 2F).

Sev reduces neuron numbers in the DG of hippocampus. Nissl staining was used to examine the numbers of DG neurons. In the control group, granule neurons exhibited round nuclei, which werelocated in the center of the perikaryon and surrounded by a pale cytoplasm (Fig. 3A). Decreased numbers of granule neurons and vacuoles were observed in the Sev group (Fig. 3B).

Sev decreases BDNF levels and inhibits PKA/CREB/BDNF signaling in the hippocampus. The level of hippocampal BDNF was measured via ELISA.BDNF levels were significantly decreased following repeated exposure to Sev (Fig. 4A). BDNF levels in the control group were7.2 $\pm 1.6 \mathrm{pg} / \mathrm{mg}$ protein, which was significantly higher compared with the Sev-treated group at $5.9 \pm 0.8 \mathrm{pg} / \mathrm{mg}$ protein. 


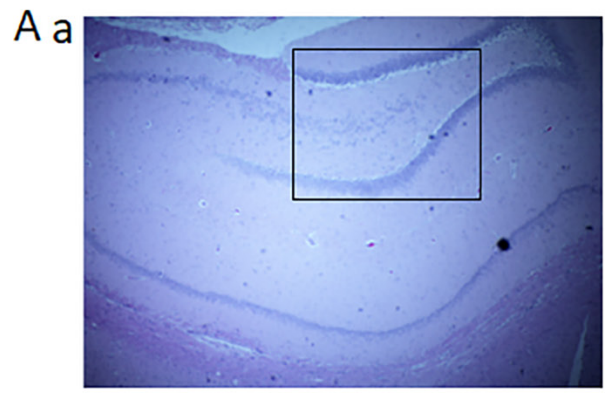

$\mathrm{Ba}$

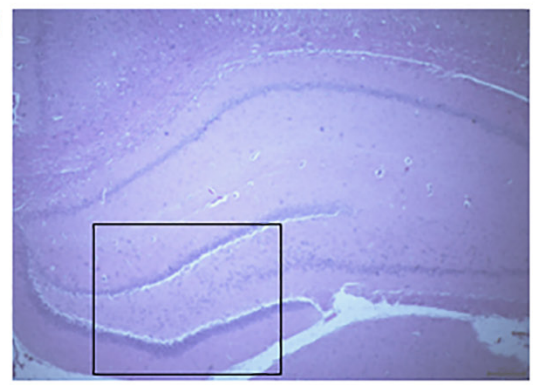

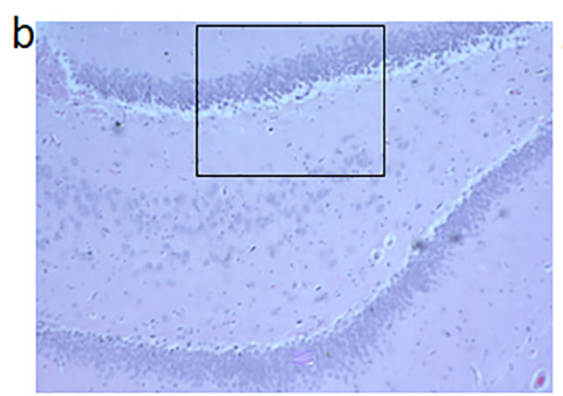

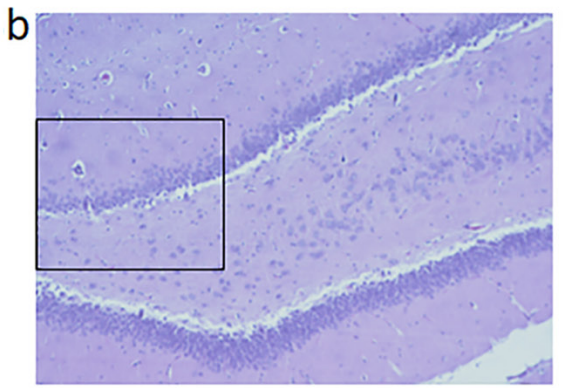

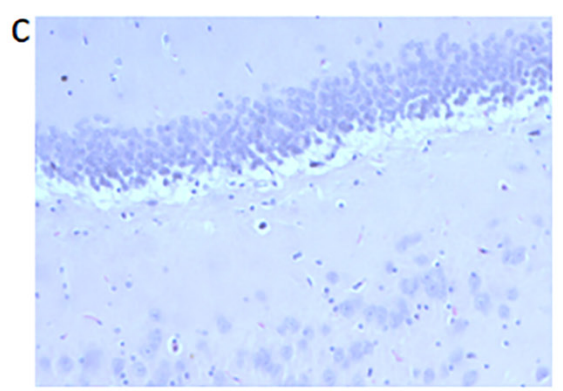

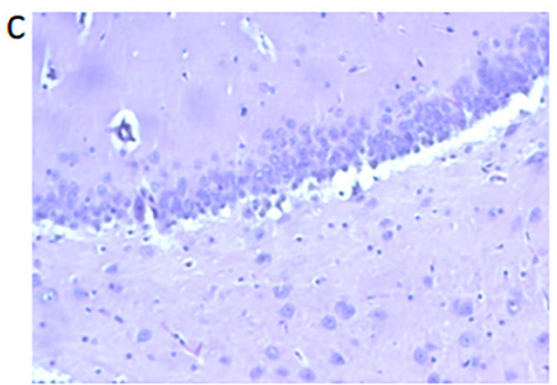

Figure 3. Different morphological profiles of granule cells visualized Nissl-stained sections in the dentate gyrus. Repeated neonatal exposure to Sev reduced the number of granule neurons in adults. (A-a) Granule neurons in the control group exhibited round nuclei, located in the center of the perikaryon and surrounded by a pale cytoplasm. (B-a) The numbers of granule neurons in the Sev groupware decreased and vacuoles were observed. A-b and B-b (magnification, x100) are magnified views of the indicated regions from A-a and B-a (magnification, x40). A-c and B-c (magnification, x200) are magnified views of indicated regions from $\mathrm{Ab}$ and $\mathrm{Bb}$. $\mathrm{n}=4$ /group. Sev, sevoflurane.

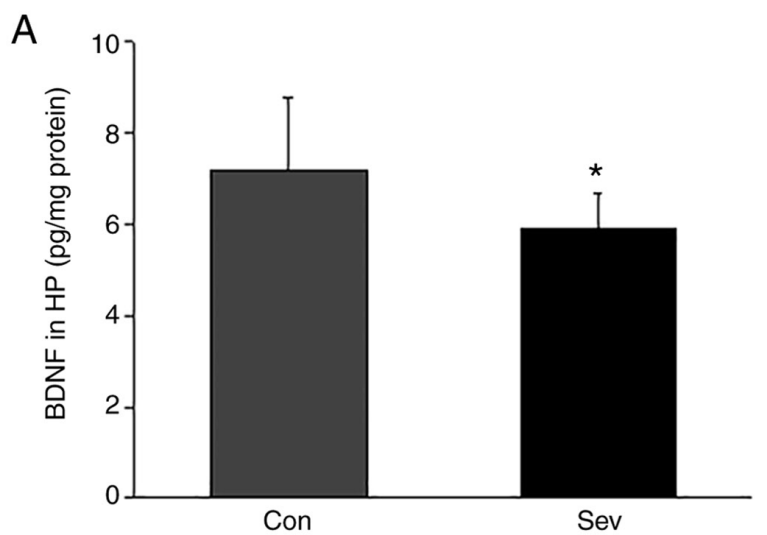

B

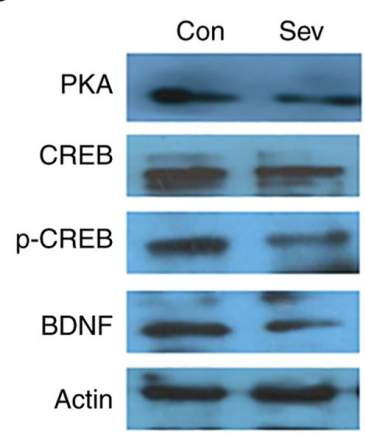

C

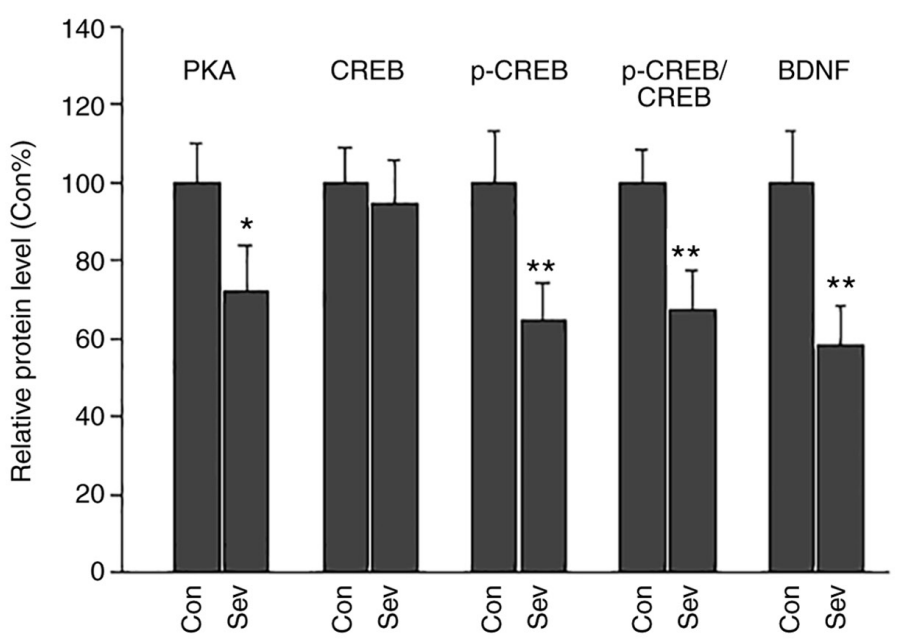

Figure 4. Effect of repeated neonatal exposure to Sev on hippocampal BDNF levels and PKA-CREB-BDNF signaling in adult rats. (A) BDNF levels in hippocampus in Con and Sev group. Repeated Sev exposure significantly decreased hippocampal BDNF levels. (B) Representative western blotting images of PKA, CREB, p-CREB and BDNF. (C) Semi-quantitative analysis of the western blotting results. Expression of PKA, p-CREB, BDNF and the p-CREB/CREB ratio was decreased in Sev group compared with the control group. $\mathrm{n}=5 /$ group. ${ }^{*} \mathrm{P}<0.05 ;{ }^{* *} \mathrm{P}<0.01$ vs. Con. Sev, sevoflurane; BDNF, brain-derived neurotrophic factor; PKA, protein kinase A; CREB, cAMP response element binding protein; p-, phosphorylated; Con, control; HP, hippocampus. 
A

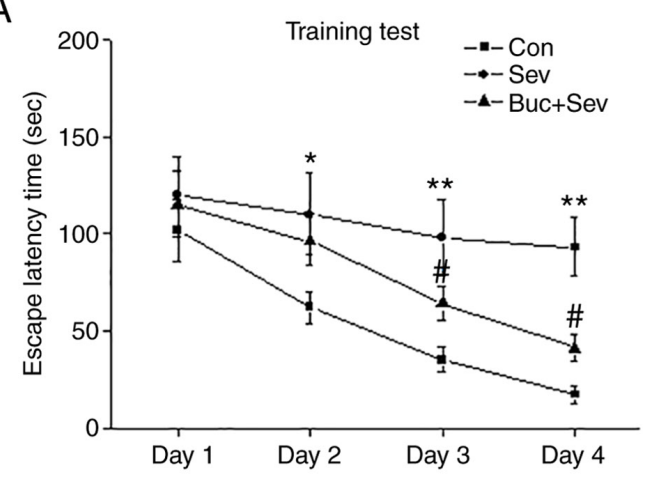

C

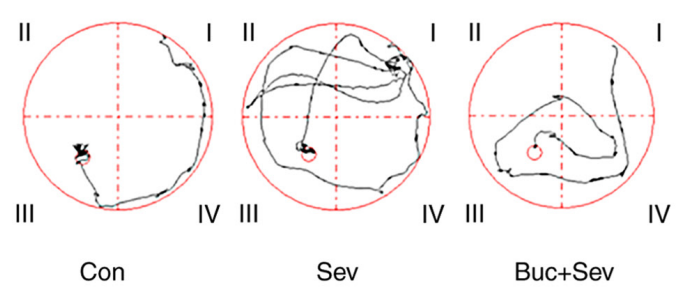

$\mathrm{E}$

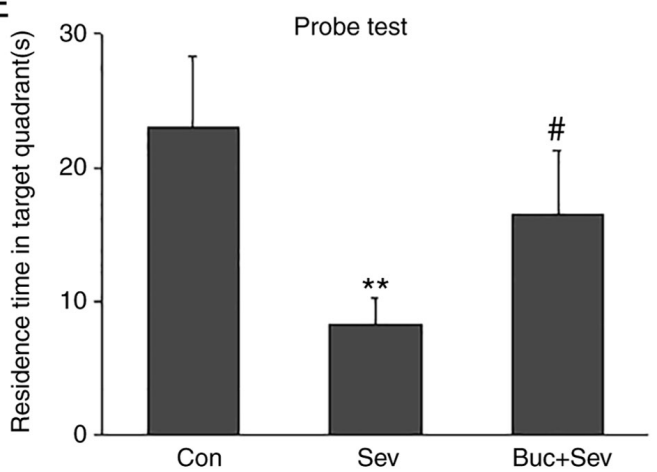

B

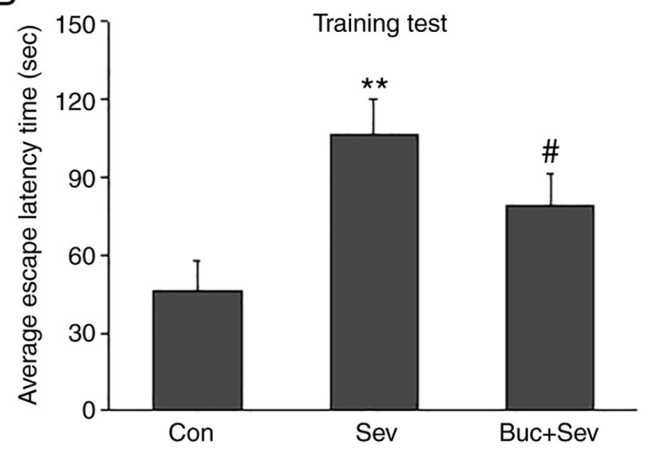

D

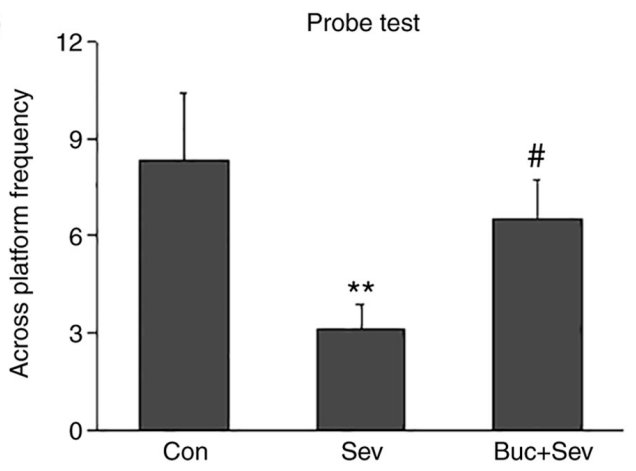

$\mathrm{F}$

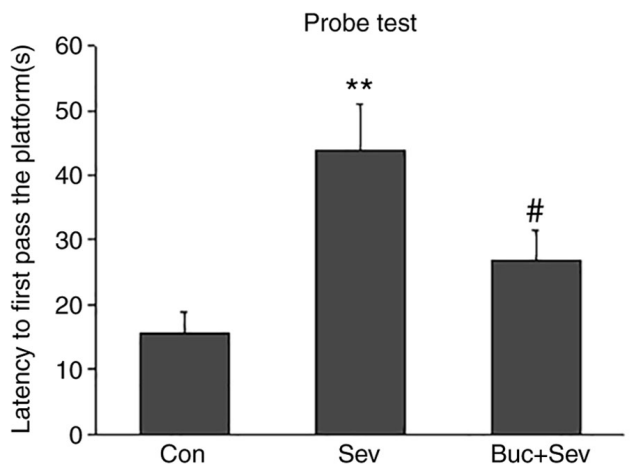

Figure 5. Buc improves the learning and memory deficit induced by Sev examined by Morris water maze tests. (A) Individual and (B) average escape latency times during the training tests. (C) Motion trails during the probe tests. (D) Across platform frequency during the probe tests. (E) Residence time in target quadrants during the probe tests. (F) Latency to first pass the platform during the probe tests. $\mathrm{n}=8$ /group. ${ }^{*} \mathrm{P}<0.05 ;{ }^{* * *} \mathrm{P}<0.01$ vs. Con. ${ }^{\text {} P} \mathrm{P}<0.05$ vs. Sev. Buc, bucladesine; Sev, sevoflurane; Con, control.

Expression of PKA, CREB, p-CREB and BDNF in the hippocampus was quantified via western blotting (Fig. 4B and C). The relative protein levels of PKA, p-CREB and BDNF in Sev group were significantly decreased compared with the Con group. CREB expression was not significantly different between the Con and Sev groups. Furthermore, the p-CREB/CREB ratio was calculated. The p-CREB/CREB ratio in Sev group was lower than that in the Con group. These results demonstrated that $\mathrm{Sev}$ inhibited the activation of the PKA/CREB/BDNF signaling pathway.

Activation of PKA-CREB signaling improves cognition and restores $B D N F$ levels. To examine the underlying pathogenesis caused by Sev, rats were treated with the PKA-selective agonist Buc. Cognition was evaluated using Morris water maze tests. In the training tests, escape latency times (Fig. 5A) and the average escape latency times (Fig. 5B) were significantly decreased in Buc-treated rats compared with only Sev-treated rats. In probe tests, the across platform frequency (Fig. 5C and D) and residence time in target quadrants (Fig. 5E) were significantly increased in Buc-treated rats compared with only Sev-treated rats. Furthermore, the latency to first pass the platform was significantly decreased in Buc-treated rats compared with rats treated solely with Sev (Fig. 5F).

Additionally, the effect of Buc on expression of PKA, CREB, p-CREB and BDNF was examined via western blotting. Buc activated PKA/CREB/BDNF signaling. Following the administration of Buc, the expression of PKA, p-CREB and BDNF in the hippocampus were notably increased compared with rats treated solely with $\mathrm{Sev}$ (Fig. 6A and B). CREB expression was not significantly different between the groups. These results indicated that the cognitive impairment caused by Sev was dependent on the $\mathrm{PKA} / \mathrm{CREB} / \mathrm{BDNF}$ pathway. 


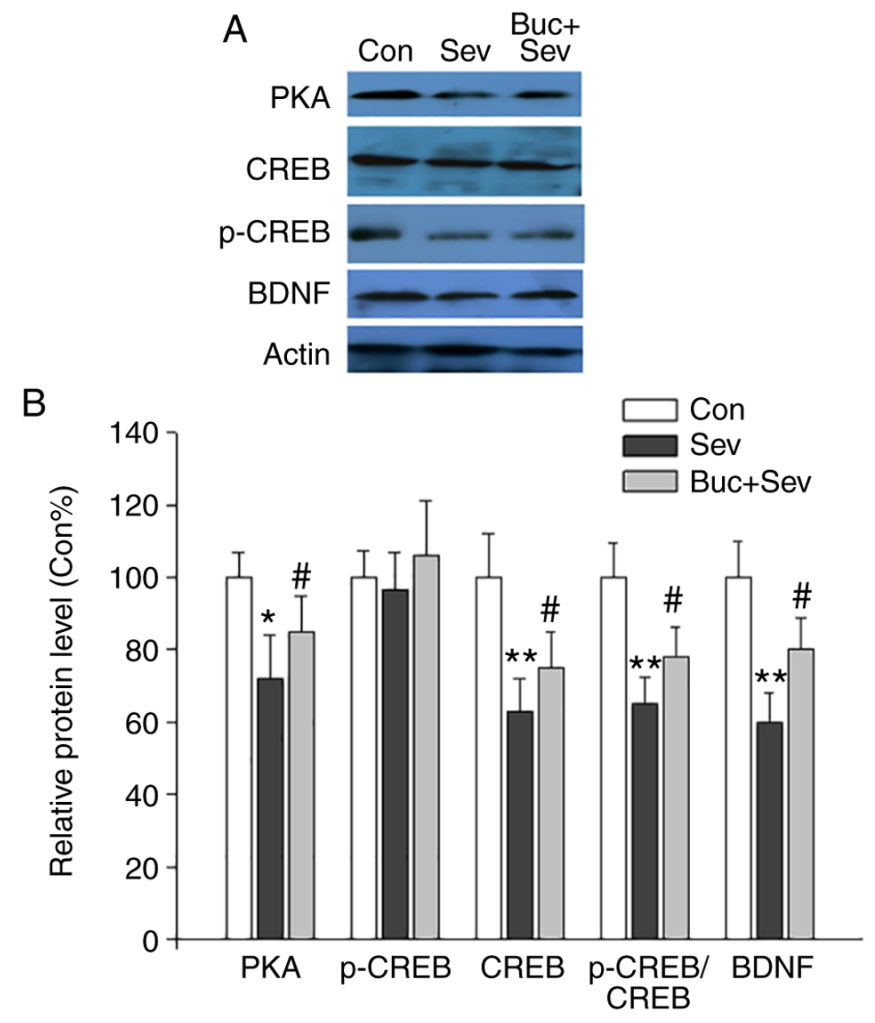

Figure 6. Buc activates the PKA-CREB-BDNF signaling pathway. (A) Representative western blotting images of PKA, CREB, p-CREB and BDNF. (B) Semi-quantitative analysis of the western blotting results. Buc upregulated the protein expression of PKA, $\mathrm{p}-\mathrm{CREB}$ and $\mathrm{BDNF}$ and the ratio of $\mathrm{p}$-CREB/CREB. $\mathrm{n}=5$ /group. ${ }^{*} \mathrm{P}<0.05 ;{ }^{*} \mathrm{P}<0.01$ vs. Con; ${ }^{\prime \prime} \mathrm{P}<0.05$ vs. Sev. Buc, bucladesine; PKA, protein kinase A; CREB, cAMP response element binding protein; $p$, phosphorylated; BDNF, brain-derived neurotrophic factor; Con, control; Sev, sevoflurane.

\section{Discussion}

Sev is a commonly used inhalational anesthetic in pediatric surgery and exhibits minimal airway reactivity and a low blood/gas partition coefficient (2). Although Sev has various advantages, it is crucial to elucidate its effects on brain development, particularly on safety issues arising from the use of anesthesia in children (20). The current study evaluated the effect of Sev on cognition in rats. The main finding of the current study was that repeated neonatal exposure to Sev induced cognitive impairment in adulthood. The results demonstrated that hippocampal BDNF levels were significantly decreased in rats treated with Sev, with the loss of granule neurons and inhibition of the PKA/CREB/BDNF signaling pathway. Cognitive impairment caused by Sev was partially reversed by the activation of the PKA/CREB/BDNF signaling pathway following the administration of the PKA agonist Buc (21). These results indicated that cognitive deficiencies caused by Sev may be restored by activation of the PKA/CREB/BDNF pathway.

Rats were treated at P6-8 days to investigate whether repeated neonatal exposure to $\mathrm{Sev}$ caused cognitive dysfunction in adult rats. The results revealed that Sev treatment in newborn rats resulted in behavioral changes in later life, as demonstrated by the Morris water maze tests. Repeated exposure to Sev caused cognitive impairment in the training and probe tests.
The DG of the hippocampus is known to serve an important role in cognitive function (22). The results demonstrated that repeated exposure to $\mathrm{Sev}$ reduced the numbers of granule cells in the DG. These reductions in cell numbers may be associated with BDNF expression. BDNF is expressed in multiple areas of the brain in mammals and is critical for neuronal survival, plasticity and morphogenesis (23). The binding of BDNF to tropomyosin receptor kinase B (TrkB) is known to activate multiple intracellular signaling pathways (24). In cultured neurons, sustained TrkB activation promotes neuronal dendritic arborization and spinogenesis, whereas transient TrkB activation facilitates dendritic growth and spine morphogenesis. In hippocampal slices, slow delivery of BDNF facilitates LTP, whereas fast application of BDNF enhances basal synaptic transmission in Schaffer collateral synapses. High-frequency stimulation of neurons converts BDNF-induced TrkB signaling from a transient to a sustained mode (25). Furthermore, Sev has been reported to down-regulate BDNF/TrkB signaling in neonatal mice (26).

CREB is a component of multiple intracellular signaling pathways and serves an important role in the nervous system. CREB modulates transcription factors via phosphorylation (27) and CREB signaling in the hippocampus is associated with emotional and cognitive behaviors (28). The phosphorylation of CREB by PKA serves a role in various nervous system diseases such as Alzheimer's disease and craniocerebral trauma $(29,30)$, and BDNF is a downstream target of PKA/CREB signaling (31). Several studies have reported that BDNF down-regulation contributes to structural damage and functional impairment in the central nervous system (32-35). The results of the current study revealed that the expression of PKA, p-CREB and BDNF was down-regulated in the hippocampus following Sev treatment. It has been hypothesized that the activation of PKA/CREB/BDNF signaling pathways may improve cognition (36). Therefore, rats were treated with Buc, a selective PKA agonist (20), and the results demonstrated that the activation of PKA significantly improved cognition in Sev-treated rats. Furthermore, when Buc was administered to rats, the expression of PKA, p-CREB and BDNF in the hippocampus was increased compared with rats treated only with Sev. In conclusion, the results of the current study indicated that $\mathrm{Sev}$ induces cognitive impairment in rats via the PKA-CREB-BDNF signaling pathway.

\section{Acknowledgements}

Not applicable.

\section{Funding}

This study was financially supported by Key Research and Development Projects in Shandong (grant no. 2018GSF118216) and Development Plan of Medical and Health Science and Technology in Shandong (grant no. 2017WS524).

\section{Availability of data and materials}

The datasets used and/or analyzed during the current study are available from the corresponding author on reasonable request. 


\section{Authors' contributions}

JZ, JR, SL, YG and PM made substantial contributions to the conception and design of the study, as well as the acquisition and analysis of data. HT and YC confirm the authenticity of all the raw data, conceived the study, performed the experiments, drafted the manuscript, revised it critically for important intellectual content and provided final approval of the version to be published. All authors read and approved the final version of the manuscript.

\section{Ethics approval and consent to participate}

Ethics approval was provided by the Animal Experimental Ethical Inspection of Laboratory Animal Center, Zhangqiu District Maternal and Child Health Care Hospital (Jinan China).

\section{Patient consent for publication}

Not applicable.

\section{Competing interests}

The authors declare that they have no competing interests.

\section{References}

1. Kim EH, Song IK, Lee JH, Kim HS, Kim HC, Yoon SH, Jang YE and Kim JT: Desflurane versus sevoflurane in pediatric anesthesia with a laryngeal mask airway: A randomized controlled trial. Medicine (Baltimore) 96: e7977, 2017.

2. Noga ML, Yarr JE and Chen PE: Evaluation of sevoflurane as an anesthetic agent for voiding cystourethrography in pediatric patients. Can Assoc Radiol J 63: 222-227, 2012.

3. Satomoto M, Sun Z, Adachi YU and Makita K: Neonatal sevoflurane exposure induces adulthood fear-induced learning disability and decreases glutamatergic neurons in the basolateral amygdala. J Neurosurg Anesthesiol 30: 59-64, 2018.

4. Karaman T, Karaman S, Doğru S, Tapar H, Şahin A and Süren M: Short-term and long-term effects of dexamethasone on cognitive dysfunction induced by sevoflurane in adult rats. Turk J Anaesthesiol Reanim 45: 158-163, 2017

5. Sun GY, Xie K, Sun ZY, Sun MY and Li N: Sevoflurane induces temporary spatial working memory deficits and synaptic ultrastructure impairments in the hippocampus of neonatal rats. Eur Rev Med Pharmacol Sci 23: 2620-2629, 2019.

6. Zheng SQ, An LX, Cheng X and Wang YJ: Sevoflurane causes neuronal apoptosis and adaptability changes of neonatal rats. Acta Anaesthesiol Scand 57: 1167-1174, 2013.

7. Andropoulos DB: Effect of anesthesia on the developing brain: Infant and fetus. Fetal Diagn Ther 43: 1-11, 2018.

8. Popova NK, Ilchibaeva TV and Naumenko VS: Neurotrophic factors (BDNF and GDNF) and the serotonergic system of the brain. Biochemistry (Mosc) 82: 308-317, 2017.

9. Leal G, Bramham CR and Duarte CB: BDNF and hippocampal synaptic plasticity. Vitam Horm 104: 153-195, 2017.

10. Tao X, Finkbeiner S, Arnold DB, Shaywitz AJ and Greenberg ME: $\mathrm{Ca}^{2+}$ influx regulates BDNF transcription by a CREB family transcription factor-dependent mechanism. Neuron 20: 709-726, 1998.

11. Yin Y, Gao D, Wang Y, Wang ZH, Wang X, Ye J, Wu D, Fang L, Pi G, Yang Y, et al: Tau accumulation induces synaptic impairment and memory deficit by calcineurin-mediated inactivation of nuclear CaMKIV/CREB signaling. Proc Natl Acad Sci USA 113: E3773-E3781, 2016.

12. West AE, Chen WG, Dalva MB, Dolmetsch RE, Kornhauser JM, Shaywitz AJ, Takasu MA, Tao X and Greenberg ME: Calcium regulation of neuronal gene expression. Proc Natl Acad Sci USA 98: 11024-11031, 2001.
13. Sharma N,Lopez DI and Nyborg JK: DNA binding and phosphorylation induce conformational alterations in the kinase-inducible domain of CREB. Implications for the mechanism of transcription function. J Biol Chem 282: 19872-19883, 2007.

14. Guo Y and Feng P: OX2R activation induces PKC-mediated ERK and CREB phosphorylation. Exp Cell Res 318: 2004-2013, 2012.

15. Al Rahim M, Nakajima A, Saigusa D, Tetsu N, Maruyama Y, Shibuya M, Yamakoshi H, Tomioka Y, Iwabuchi Y, Ohizumi Y and Yamakuni T: 4'-Demethylnobiletin, a bioactive metabolite of nobiletin enhancing PKA/ERK/CREB signaling, rescues learning impairment associated with NMDA receptor antagonism via stimulation of the ERK cascade. Biochemistry 48: 7713-7721, 2009

16. Bi C, Cai Q, Shan Y, Yang F, Sun S, Wu X and Liu H: Sevoflurane induces neurotoxicity in the developing rat hippocampus by upregulating connexin 43 via the JNK/c-Jun/AP-1 pathway. Biomed Pharmacother 108: 1469-1476, 2018.

17. Sharifzadeh M, Zamanian AR, Gholizadeh S, Tabrizian K, Etminani M, Khalaj S, Zarrindast MR and Roghani A: Post-training intrahippocampal infusion of nicotine-bucladesine combination causes a synergistic enhancement effect on spatial memory retention in rats. Eur J Pharmacol 562: 212-220, 2007.

18. Hosseini-Zare MS, Salehi F, Seyedi SY, Azami K, Ghadiri T, Mobasseri M, Gholizadeh S, Beyer C and Sharifzadeh M: Effects of pentoxifylline and H-89 on epileptogenic activity of bucladesine in pentylenetetrazol-treated mice. Eur J Pharmacol 670: 464-470, 2011

19. Yang Y, Wang L, Wu Y, Su D, Wang N, Wang J, Shi C, Lv L and Zhang S: Tanshinol suppresses inflammatory factors in a rat model of vascular dementia and protects LPS-treated neurons via the MST1-FOXO3 signaling pathway. Brain Res 1646: 304-314, 2016.

20. Yu M, Han C, Zhou Q, Liu C and Ding Z: Clinical effects of sevoflurane anesthesia induction with a portable inhalational anesthetic circuit in pediatric patients. Arch Med Sci 11: 796-800, 2015.

21. Salehi F, Hosseini-Zare MS, Aghajani H, Seyedi SY, Hosseini-Zare MS and Sharifzadeh M: Effect of bucladesine, pentoxifylline, and $\mathrm{H}-89$ as cyclic adenosine monophosphate analog, phosphodiesterase, and protein kinase A inhibitor on acute pain. Fundam Clin Pharmacol 31: 411-419, 2017.

22. Brickman AM, Khan UA, Provenzano FA, Yeung LK, Suzuki W, Schroeter H, Wall M, Sloan RP and Small SA: Enhancing dentate gyrus function with dietary flavanols improves cognition in older adults. Nat Neurosci 17: 1798-1803, 2014.

23. Numakawa T, Suzuki S, Kumamaru E, Adachi N, Richards M and Kunugi H: BDNF function and intracellular signaling in neurons. Histol Histopathol 25: 237-258, 2010.

24. Andero R, Choi DC and Ressler KJ: BDNF-TrkB receptor regulation of distributed adult neural plasticity, memory formation, and psychiatric disorders. Prog Mol Biol Transl Sci 122: 169-192, 2014.

25. Guo W, Nagappan G and Lu B: Differential effects of transient and sustained activation of BDNF-TrkB signaling. Dev Neurobiol 78: 647-659, 2018

26. Ding ML, Ma H, Man YG and Lv HY: Protective effects of a green tea polyphenol, epigallocatechin-3-gallate, against sevoflurane-induced neuronal apoptosis involve regulation of $\mathrm{CREB} / \mathrm{BDNF} / \mathrm{TrkB}$ and PI3K/Akt/mTOR signalling pathways in neonatal mice. Can J Physiol Pharmacol 95: 1396-1405, 2017.

27. Wang L, Hu XH, Huang ZX, Nie Q, Chen ZG, Xiang JW, Qi RL, Yang TH, Xiao Y, Qing WJ, et al: Regulation of CREB functions by phosphorylation and sumoylation in nervous and visual systems. Curr Mol Med 16: 885-892, 2017.

28. Dong W, Xu D, Hu Z, He X, Guo Z, Jiao Z, Yu Y and Wang H: Low-functional programming of the CREB/BDNF/TrkB pathway mediates cognitive impairment in male offspring after prenatal dexamethasone exposure. Toxicol Lett 283: 1-12, 2018.

29. Luo Y,Kuang S,LiH,RanDand Yang J:cAMP/PKA-CREB-BDNF signaling pathway in hippocampus mediates cyclooxygenase 2 -induced learning/memory deficits of rats subjected to chronic unpredictable mild stress. Oncotarget 8: 35558-35572, 2017.

30. Liu H, Jin X, Yin X, Jin N, Liu F and Qian W: PKA-CREB signaling suppresses tau transcription. J Alzheimers Dis 46: 239-248, 2015

31. Han C, Yang Y, Ruan S, Guo L, Zhang X and Guan Q: The predictive value of serum $p$-CREB level on secondary cognitive impairment in patients with mild-to-moderate craniocerebral trauma. Neurosurg Rev 42: 715-720, 2019. 
32. Rosa $E$ and Fahnestock M: CREB expression mediates amyloid $\beta$-induced basal BDNF downregulation. Neurobiol Aging 36: 2406-2413, 2015.

33. Lu B, Nagappan G and Lu Y: BDNF and synaptic plasticity, cognitive function, and dysfunction. Handb Exp Pharmacol 220: 223-250, 2014.

34. Rahmani M, Rahmani F and Rezaei N: The brain-derived neurotrophic factor: Missing link between sleep deprivation, insomnia, and depression. Neurochem Res 45: 221-231, 2020.

35. Wang R and Holsinger RMD: Exercise-induced brain-derived neurotrophic factor expression: Therapeutic implications for Alzheimer's dementia. Ageing Res Rev 48: 109-121, 2018.
36. Shin MS, Kim TW, Park SS, Ko IG, Kim CJ, Kim M, Roh SY, Kim KT and Kim KH: Long-term surgical and chemical castration deteriorates memory function through downregulation of PKA/CREB/BDNF and c-Raf/MEK/ERK pathways in hippocampus. Int Neurourol J 23: 116-124, 2019.

(i) (9) This work is licensed under a Creative Commons caY AO NO Attribution-NonCommercial-NoDerivatives 4.0 International (CC BY-NC-ND 4.0) License. 